\title{
An Optimization Problem of Boundary Type for Cooperative Hyperbolic Systems Involving Schrödinger Operator
}

\author{
Ahlam Hasan Qamlo \\ Department of Mathematics, Faculty of Applied sciences, Umm AL-Qura University, Makkah, KSA \\ Email: drahqamlo@yahoo.com
}

Received 16 September 2014; revised 10 October 2014; accepted 25 October 2014

Copyright (C) 2014 by author and Scientific Research Publishing Inc.

This work is licensed under the Creative Commons Attribution International License (CC BY).

http://creativecommons.org/licenses/by/4.0/

(c) (i) Open Access

\begin{abstract}
In this paper, we consider cooperative hyperbolic systems involving Schrödinger operator defined on $R^{n}$. First we prove the existence and uniqueness of the state for these systems. Then we find the necessary and sufficient conditions of optimal control for such systems of the boundary type. We also find the necessary and sufficient conditions of optimal control for same systems when the observation is on the boundary.
\end{abstract}

\section{Keywords}

Hyperbolic Systems, Schrödinger Operator, Boundary Control Problem, Boundary Observation, Cooperative

\section{Introduction}

The optimal control problems of distributed systems involving Schrödinger operator have been widely discussed in many papers. One of the first studies was introduced by Serag [1], which discusses $2 \times 2$ cooperative systems of elliptic operator. Further research in this area developed the problem by studying different operator types (elliptic, parabolic, or hyperbolic) or higher system degree as in [2]-[6]. Many boundary control problems have been introduced in [7]-[10].

In [3], we discussed distributed control problem for $2 \times 2$ cooperative hyperbolic systems involving Schrödinger operator.

Here, using the theory of [11], we consider the following $2 \times 2$ cooperative hyperbolic systems involving Schrödinger operator: 


$$
\begin{cases}\frac{\partial^{2} y_{1}(x)}{\partial t^{2}}+(-\Delta+q) y_{1}=a y_{1}+b y_{2}+f_{1}(x, t), & \text { in } Q, \\ \frac{\partial^{2} y_{2}(x)}{\partial t^{2}}+(-\Delta+q) y_{2}=c y_{1}+d y_{2}+f_{2}(x, t), & \text { in } Q, \\ y_{1}, y_{2} \rightarrow 0 \text { as } \quad|x| \rightarrow \infty, & \\ \left.\frac{\partial y_{1}}{\partial v}\right|_{\Sigma}=\left.\frac{\partial y_{2}}{\partial v}\right|_{\Sigma}=0, & \text { in } R^{n}, \\ y_{1}(x, 0)=y_{1,0}(x), \quad y_{2}(x, 0)=y_{2,0}(x), & \text { in } R^{n} . \\ \frac{\partial y_{1}(x, 0)}{\partial t}=y_{1,1}(x), \quad \frac{\partial y_{2}(x, 0)}{\partial t}=y_{2,1}(x), & \end{cases}
$$

with $y_{1}, y_{2} \in L^{2}\left(0, T ; V_{q}\left(R^{n}\right)\right), \frac{\partial y_{1}}{\partial t}, \frac{\partial y_{2}}{\partial t} \in L^{2}(Q)$.

where $a, b, c$ and $d$ are given numbers such that $b, c>0$,

i.e. the system (1) is called cooperative

$q(x)$ is a positive function and tending to $\infty$ at infinity,

and $\left.Q=R^{n} \times\right] 0, T[$ with boundary $\Sigma=\Gamma \times] 0, T[$.

The model of the system (1) is given by:

$$
B(t) y(x)=B(t)\left(y_{1}(x), y_{2}(x)\right)=\left(\frac{\partial^{2} y_{1}}{\partial t^{2}}+(-\Delta+q) y_{1}-a y_{1}-b y_{2}, \frac{\partial^{2} y_{2}}{\partial t^{2}}+(-\Delta+q) y_{2}-c y_{1}-d y_{2}\right)
$$

since $A(t) y(x)=\left((-\Delta+q) y_{1}-a y_{1}-b y_{2},(-\Delta+q) y_{2}-c y_{1}-d y_{2}\right), \quad A(t) y \in\left(V_{q}^{\prime}\left(R^{n}\right)\right)^{2}$.

We first prove the existence and uniqueness of the state for these systems, then we introduce the optimality conditions of boundary control, we also discuss them when the observation is on the boundary.

\section{Some Concepts and Results}

Here we shall consider some results about the following eigenvalue problem which introduced in [1] and [12]:

$$
\left\{\begin{array}{l}
(-\Delta+q) \phi=\lambda(q) \phi \quad \text { in } R^{n} \\
\phi(x) \rightarrow 0 \text { as }|x| \rightarrow \infty, \quad \phi \succ 0
\end{array}\right.
$$

The associated space is $V_{q}\left(R^{n}\right)$, with respect to the norm:

$$
\|y\|_{q}=\left(\int_{R^{n}}\left[|\nabla y|^{2}+q|y|^{2}\right] \mathrm{d} x\right)^{1 / 2}
$$

Since the imbedding of $V_{q}\left(R^{n}\right)$ into $L^{2}\left(R^{n}\right)$ is compact, then the operator $(-\Delta+q)$ considered as an Operator in $L^{2}\left(R^{n}\right)$ is positive self-adjoint with compact inverse. Hence its spectrum consists of an infinite sequence of positive eigenvalues, tending to infinity; moreover the smallest one which is called the principal eigenvalue denoted by $\lambda(q)$ is simple and is associated with an eigenfunction which does not change sign in $R^{n}$. It is characterized by:

$$
\lambda(q) \int_{R^{n}}|y|^{2} \mathrm{~d} x \leq \int_{R^{n}}\left[|\nabla y|^{2}+q|y|^{2}\right] \mathrm{d} x \quad \forall y \in V_{q}\left(R^{n}\right)
$$

We have:

$$
V_{q}\left(R^{n}\right) \times V_{q}\left(R^{n}\right) \subseteq L^{2}\left(R^{n}\right) \times L^{2}\left(R^{n}\right) \subseteq V_{q}^{\prime}\left(R^{n}\right) \times V_{q}^{\prime}\left(R^{n}\right)
$$


which is continuous and compact.

Let us introduce the space $L^{2}\left(0, T ; V_{q}\left(R^{n}\right)\right)$ of measurable function $t \rightarrow f(t)$ which is defined on open interval $(0, T)$ and the variable $t \in(0, T), T<\infty$ denotes the time.

On $(0, T)$ with Lebesgue measure $\mathrm{d} t$ we have the norm:

$$
\|f(t)\|_{L^{2}\left(0, T ; V_{q}\left(R^{n}\right)\right)}=\left(\int_{(0, T)}\|f(t)\|_{V_{q}\left(R^{n}\right)}^{2} \mathrm{~d} t\right)^{1 / 2} \prec \infty
$$

and the scalar product

$$
(f(t), g(t))_{L^{2}\left(0, T ; V_{q}\left(R^{n}\right)\right)}=\int_{(0, T)}(f(t), g(t))_{V_{q}\left(R^{n}\right)} \mathrm{d} t,
$$

the space $L^{2}\left(0, T ; V_{q}\left(R^{n}\right)\right)$ with the scalar product and the norm above is a Hilbert space.

Analogously, we can define the spaces $L^{2}\left(0, T ; L^{2}\left(R^{n}\right)\right)=L^{2}(Q)$,

with the scalar product:

$$
(f(t), g(t))_{L^{2}(Q)}=\int_{(0, T)}(f(t), g(t))_{L^{2}\left(R^{n}\right)} \mathrm{d} t=\int_{Q} f(t) \cdot g(t) \mathrm{d} x \mathrm{~d} t
$$

then we have:

$$
L^{2}\left(0, T ; V_{q}\left(R^{n}\right)\right) \times L^{2}\left(0, T ; V_{q}\left(R^{n}\right)\right) \subseteq L^{2}(Q) \times L^{2}(Q) \subseteq L^{2}\left(0, T ; V_{q}^{\prime}\left(R^{n}\right)\right) \times L^{2}\left(0, T ; V_{q}^{\prime}\left(R^{n}\right)\right)
$$

\section{The Existence and Uniqueness for the State of the System (1)}

We have the bilinear form:

$$
\begin{aligned}
& \pi(t ; y, \psi)= \frac{1}{b} \int_{R^{n}}\left[\nabla y_{1} \nabla \psi_{1}+q y_{1} \psi_{1}\right] \mathrm{d} x+\frac{1}{c} \int_{R^{n}}\left[\nabla y_{2} \nabla \psi_{2}+q y_{2} \psi_{2}\right] \mathrm{d} x-\int_{R^{n}} y_{1} \psi_{2} \mathrm{~d} x-\frac{d}{c} \int_{R^{n}} y_{2} \psi_{2} \mathrm{~d} x \\
&-\frac{a}{b} \int_{R^{n}} y_{1} \psi_{1} \mathrm{~d} x-\int_{R^{n}} y_{2} \psi_{1} \mathrm{~d} x, \\
& y=y_{1}, y_{2}, \quad \psi=\left(\psi_{1}, \psi_{2}\right) \in\left(V_{q}\left(R^{n}\right)\right)^{2} .
\end{aligned}
$$

For all $y, \psi \in\left(V_{q}\left(R^{n}\right)\right)^{2}$ the function $t \rightarrow \pi(t ; y, \psi)$ is measurable on $(0, T)$.

The coerciveness condition of the bilinear form (7) in $\left(V_{q}\left(R^{n}\right)\right)^{2}$ has been proved by Serag [1], by using the conditions for having the maximum principle for cooperative system (1) which have been obtained by Fleckinger [13], and take the form:

$$
\left\{\begin{array}{l}
a \prec \lambda(q), \quad d \prec \lambda(q), \\
(\lambda(q)-a)(\lambda(q)-d) \succ b c
\end{array}\right.
$$

that means:

$$
\pi(t ; y, y) \geq C\left(\left\|y_{1}\right\|_{q, m}^{2}+\left\|y_{2}\right\|_{q, m}^{2}\right), \quad C \succ 0
$$

Theorem (3.1):

Under the hypotheses (2) and (9), if $f_{1}, f_{2} \in L^{2}\left(0, T ; V_{q}^{\prime}\left(R^{n}\right)\right), y_{1,0}(x), y_{2,0}(x) \in V_{q}\left(R^{n}\right)$ and $y_{1,1}(x)$, $y_{2,1}(x) \in L\left(R^{n}\right)$, then there exists a unique solution: $y=\left\{y_{1}, y_{2}\right\} \in\left(L^{2}\left(0, T ; V_{q}\left(R^{n}\right)\right)\right)^{2}$ for system (1). 


\section{Proof:}

Let $\psi \rightarrow L(\psi)$ be a continuous linear form defined on $\left(V_{q}\left(R^{n}\right)\right)^{2}$ by:

$$
\begin{aligned}
L(\psi)= & \frac{1}{b} \int_{Q} f_{1}(x, t) \psi_{1}(x) \mathrm{d} x \mathrm{~d} t+\frac{1}{c} \int_{Q} f_{2}(x, t) \psi_{2}(x) \mathrm{d} x \mathrm{~d} t \\
& +\frac{1}{b} \int_{R^{n}} y_{1,1}(x) \frac{\partial \psi_{1}(x, 0)}{\partial t} \mathrm{~d} x+\frac{1}{c} \int_{R^{n}} y_{2,1}(x) \frac{\partial \psi_{2}(x, 0)}{\partial t} \mathrm{~d} x, \quad \forall\left(\psi_{1}, \psi_{2}\right) \in\left(V_{q}\left(R^{n}\right)\right)^{2} .
\end{aligned}
$$

then by Lax-Milgram lemma, there exists a unique element $y=\left(y_{1}, y_{2}\right) \in\left(V_{q}\left(R^{n}\right)\right)^{2}$ such that:

$$
\pi(t ; y, \psi)=L(\psi), \quad \forall \psi=\left(\psi_{1}, \psi_{2}\right) \in\left(V_{q}\left(R^{n}\right)\right)^{2}
$$

Now, let us multiply both sides of first equation of system (1) by $\frac{1}{b} \psi_{1}(x)$, and the second equation by: $\frac{1}{c} \psi_{2}(x)$ then integration over $Q$, we have:

$$
\begin{aligned}
& \frac{1}{b} \int_{Q}\left[\frac{\partial^{2} y_{1}(x)}{\partial t^{2}}+(-\Delta+q) y_{1}-a y_{1}-b y_{2}\right] \psi_{1} \mathrm{~d} x \mathrm{~d} t=\frac{1}{b} \int_{Q} f_{1}(x, t) \psi_{1} \mathrm{~d} x \mathrm{~d} t \\
& \frac{1}{c} \int_{Q}\left[\frac{\partial^{2} y_{2}(x)}{\partial t^{2}}+(-\Delta+q) y_{2}-c y_{1}-d y_{2}\right] \psi_{2} \mathrm{~d} x \mathrm{~d} t=\frac{1}{c} \int_{Q} f_{2}(x, t) \psi_{2} \mathrm{~d} x \mathrm{~d} t
\end{aligned}
$$

By applying Green's formula:

$$
\begin{aligned}
& \frac{1}{b} \int_{R^{n}} \frac{\partial y_{1}(x, 0)}{\partial t} \frac{\partial \psi_{1}(x, 0)}{\partial t} \mathrm{~d} x+\frac{1}{b} \int_{\Sigma} \psi_{1} \frac{\partial y_{1}}{\partial t} \nu \mathrm{d} \Sigma+\frac{1}{b} \int_{Q} \nabla y_{1} \nabla \psi_{1} \mathrm{~d} x \mathrm{~d} t-\frac{1}{b} \int_{\Sigma} \psi_{1} \frac{\partial y_{1}}{\partial v_{A}} \mathrm{~d} \Sigma+\int_{Q}\left(\frac{q}{b} y_{1}-\frac{a}{b} y_{1}-y_{2}\right) \psi_{1} \mathrm{~d} x \mathrm{~d} t \\
& \quad=\frac{1}{b} \int_{Q} f_{1}(x, t) \psi_{1} \mathrm{~d} x \mathrm{~d} t, \\
& \frac{1}{c} \int_{R^{n}} \frac{\partial y_{2}(x, 0)}{\partial t} \frac{\partial \psi_{2}(x, 0)}{\partial t} \mathrm{~d} x+\frac{1}{c} \int_{\Sigma} \psi_{2} \frac{\partial y_{2}}{\partial t} \nu \mathrm{d} \Sigma+\frac{1}{c} \int_{Q} \nabla y_{2} \nabla \psi_{2} \mathrm{~d} x \mathrm{~d} t-\frac{1}{c} \int_{\Sigma} \psi_{2} \frac{\partial y_{2}}{\partial v_{A}} \mathrm{~d} \Sigma+\int_{Q}\left(\frac{q}{c} y_{2}-y_{1}-\frac{d}{c} y_{2}\right) \psi_{1} \mathrm{~d} x \mathrm{~d} t \\
& \quad=\frac{1}{c} \int_{Q} f_{2}(x, t) \psi_{c} \mathrm{~d} x \mathrm{~d} t,
\end{aligned}
$$

By sum the two equations we get:

$$
\begin{aligned}
& \frac{1}{b} \int_{R^{n}} \frac{\partial y_{1}(x, 0)}{\partial t} \frac{\partial \psi_{1}(x, 0)}{\partial t} \mathrm{~d} x+\frac{1}{b} \int_{\Sigma} \psi_{1} \frac{\partial y_{1}}{\partial t} \nu \mathrm{d} \Sigma-\frac{1}{b} \int_{\Sigma} \psi_{1} \frac{\partial y_{1}}{\partial v_{A}} \mathrm{~d} \Sigma+\frac{1}{C} \int_{R^{n}} \frac{\partial y_{2}(x, 0)}{\partial t} \frac{\partial \psi_{2}(x, 0)}{\partial t} \mathrm{~d} x+\frac{1}{C} \int_{\Sigma} \psi_{2} \frac{\partial y_{2}}{\partial t} \nu \mathrm{d} \Sigma \\
& -\frac{1}{C} \int_{\Sigma} \psi_{2} \frac{\partial y_{2}}{\partial v_{A}} \mathrm{~d} \Sigma=\frac{1}{b} \int_{R^{n}} y_{1,1}(x) \frac{\partial \psi_{1}(x, 0)}{\partial t} \mathrm{~d} x+\frac{1}{C} \int_{R^{n}} y_{2,1}(x) \frac{\partial \psi_{2}(x, 0)}{\partial t} \mathrm{~d} x,
\end{aligned}
$$

by comparing the previous equation with (7), (10) and (11) we deduce that:

$$
\begin{gathered}
\left.\frac{\partial y_{1}}{\partial v}\right|_{\Sigma}=\left.\frac{\partial y_{2}}{\partial v}\right|_{\Sigma}=0 \\
\frac{\partial y_{1}(x, 0)}{\partial t}=y_{1,1}(x), \quad \frac{\partial y_{2}(x, 0)}{\partial t}=y_{2,1}(x) \quad \text { in } R^{n}
\end{gathered}
$$

then the proof is complete.

\section{Formulation of the Control Problem}

The space $L^{2}(\Sigma) \times L^{2}(\Sigma)$ is the space of controls. For a control $u=\left(u_{1}, u_{2}\right) \in\left(L_{2}(\Sigma)\right)^{2}$, the state 
$y(u)=\left(y_{1}(u), y_{2}(u)\right) \in\left(L^{2}\left(0, T ; V_{q}\left(R^{n}\right)\right)\right)^{2}$ of the system is given by the solution of

$$
\begin{cases}\frac{\partial^{2} y_{1}(u)}{\partial t^{2}}+(-\Delta+q) y_{1}(u)=a y_{1}(u)+b y_{2}(u)+f_{1} & \text { in } Q, \\ \frac{\partial^{2} y_{2}(u)}{\partial t^{2}}+(-\Delta+q) y_{2}(u)=c y_{1}(u)+d y_{2}(u)+f_{2} & \text { in } Q, \\ y_{1}, y_{2} \rightarrow 0 \text { as } \quad|x| \rightarrow \infty, & \\ \left.\frac{\partial y_{1}(u)}{\partial v}\right|_{\Sigma}=u_{1},\left.\quad \frac{\partial y_{2}(u)}{\partial v}\right|_{\Sigma}=u_{2}, & \text { in } R^{n}, \\ y_{1}(x, 0, u)=y_{1,0}(x), \quad y_{2}(x, 0, u)=y_{2,0}(x) & \text { in } R^{n} . \\ \frac{\partial y_{1}(x, 0, u)}{\partial t}=y_{1,1}(x), \quad \frac{\partial y_{2}(x, 0, u)}{\partial t}=y_{2,1}(x) & \end{cases}
$$

with $y_{1}(u), y_{2}(u) \in L^{2}\left(0, T ; V_{q}\left(R^{n}\right)\right), \frac{\partial y_{1}(u)}{\partial t}, \frac{\partial y_{2}(u)}{\partial t} \in L^{2}(Q)$.

The observation equation is given by $z(u)=\left(z_{1}(u), z_{2}(u)\right)=y(u)=\left(y_{1}(u), y_{2}(u)\right)$.

For a given $z_{d}=\left(z_{d 1}, z_{d 2}\right) \in\left(L^{2}(Q)\right)^{2}$, the cost function is given by:

$$
J(v)=\left\|y_{1}(v)-z_{d 1}\right\|_{L^{2}(Q)}^{2}+\left\|y_{2}(v)-z_{d 2}\right\|_{L^{2}(Q)}^{2}+(N v, v)_{\left(L^{2}(\Sigma)\right)^{2}} .
$$

where $N \in L\left(\left(L^{2}(\Sigma)\right)^{2},\left(L^{2}(\Sigma)\right)^{2}\right)$ is hermitian positive definite operator:

$$
(N u, u)_{\left(L^{2}(\Sigma)\right)^{2}} \geq \gamma\|u\|_{\left(L^{2}(\Sigma)\right)^{2}}^{2}, \quad \gamma \succ 0
$$

The control problem then is to find $u=\left\{u_{1}, u_{2}\right\} \in U_{a d}$ such that $J(u) \leq J(v)$, where $U_{a d}$ is a closed convex subset of $\left(L^{2}(\Sigma)\right)^{2}$.

Since the cost function (14) can be written as (see [11]):

$$
J(v)=a(v, v)-2 L(v)+\left\|y(0)-z_{d}\right\|_{\left(L^{2}(Q)\right)^{2}}^{2}
$$

where $a(v, v)$ is a continuous coercive bilinear form and $L(v)$ is a continuous linear form on $\left(L^{2}(\Sigma)\right)^{2}$. Then there exists a unique optimal control $u \in U_{a d}$ such that $J(u)=\inf J(v)$ for all $v \in U_{a d}$ by using the general theory of Lions [11]. Moreover, we have the following theorem which gives the necessary and sufficient conditions of optimality:

Theorem (4.1):

Assume that (9) and (14) hold. If the cost function is given by (13), the optimal control $u=\left(u_{1}, u_{2}\right) \in\left(L_{2}(\Sigma)\right)^{2}$ is then characterized by the following equations and inequalities:

$$
\begin{cases}\frac{\partial^{2} p_{1}(u)}{\partial t^{2}}+(-\Delta+q) p_{1}(u)-a p_{1}(u)-c p_{2}(u)=y_{1}(u)-z_{d 1} & \text { in } Q, \\ \frac{\partial^{2} p_{2}(u)}{\partial t^{2}}+(-\Delta+q) p_{2}(u)-b p_{1}(u)-d p_{2}(u)=y_{2}(u)-z_{d 2} & \text { in } Q, \\ p_{1}, p_{2} \rightarrow 0 \text { as }|x| \rightarrow \infty, & \\ \left.\frac{\partial p_{1}(u)}{\partial v}\right|_{\Sigma}=0,\left.\quad \frac{\partial p_{2}(u)}{\partial v}\right|_{\Sigma}=0, & \text { in } R^{n}, \\ p_{1}(x, T, u)=p_{2}(x, T, u)=0 & \text { in } R^{n} . \\ \frac{\partial p_{1}(x, T, u)}{\partial t}=\frac{\partial p_{2}(x, T, u)}{\partial t}=0 & \end{cases}
$$


with $p_{1}(u), p_{2}(u) \in L^{2}\left(0, T ; V_{q}\left(R^{n}\right)\right), \frac{\partial p_{1}(u)}{\partial t}, \frac{\partial p_{2}(u)}{\partial t} \in L^{2}(Q)$

$$
(p(u)+N u, v-u)_{\left(L^{2}(\Sigma)\right)^{2}} \geq 0, \quad \forall v=\left(v_{1}, v_{2}\right) \in U_{a d}
$$

together with (12), where $p(u)=\left(p_{1}(u), p_{2}(u)\right)$ is the adjoint state.

Proof:

The optimal control $u=\left(u_{1}, u_{2}\right) \in\left(L_{2}(\Sigma)\right)^{2}$ is characterized by [11]

$$
J^{\prime}(u)(v-u) \geq 0, \quad \forall v \in U_{a d},
$$

Which is equivalent to:

$$
\left(y(u)-z_{d}, y(v)-y(u)\right)_{\left(L^{2}(Q)\right)^{2}}+(N u, v-u)_{\left(L^{2}(\Sigma)\right)^{2}} \geq 0
$$

i.e.

$$
\left(y_{1}(u)-z_{d 1}, y_{1}(v)-y_{1}(u)\right)_{L^{2}(Q)}+\left(y_{2}(u)-z_{d 2}, y_{2}(v)-y_{2}(u)\right)_{L^{2}(Q)}+(N u, v-u)_{\left(L^{2}(\Sigma)\right)^{2}} \geq 0
$$

this inequality can be written as:

$$
\int_{0}^{T}\left[\left(y_{1}(u)-z_{d 1}, y_{1}(v)-y_{1}(u)\right)_{L^{2}\left(R^{n}\right)}+\left(y_{2}(u)-z_{d 2}, y_{2}(v)-y_{2}(u)\right)_{L^{2}\left(R^{n}\right)}\right] \mathrm{d} t+(N u, v-u)_{\left(L^{2}(\Sigma)\right)^{2}} \geq 0
$$

Now, since:

$$
\begin{aligned}
(p, B y)_{\left(L^{2}(Q)\right)^{2}=} & \int_{0}^{T}\left(p_{1}(u), \frac{\partial^{2} y_{1}(u)}{\partial t^{2}}+(-\Delta+q) y_{1}(u)-a y_{1}(u)-b y_{2}(u)\right)_{L^{2}\left(R^{n}\right)} \mathrm{d} t \\
& +\int_{0}^{T}\left(p_{2}(u), \frac{\partial^{2} y_{2}(u)}{\partial t^{2}}+(-\Delta+q) y_{2}(u)-c y_{1}(u)-d y_{2}(u)\right)_{L^{2}\left(R^{n}\right)} \mathrm{d} t .
\end{aligned}
$$

where

$$
\begin{aligned}
B y(u) & =B\left(y_{1}(u), y_{2}(u)\right) \\
& =\left(\frac{\partial^{2} y_{1}(u)}{\partial t^{2}}+(-\Delta+q) y_{1}(u)-a y_{1}(u)-b y_{2}(u), \frac{\partial^{2} y_{2}(u)}{\partial t^{2}}+(-\Delta+q) y_{2}(u)-c y_{1}(u)-d y_{2}(u)\right) .
\end{aligned}
$$

by using Green formula and (12), we have:

$$
\begin{aligned}
(p, B y)_{\left(L^{2}(Q)\right)^{2}=} & \int_{0}^{T}\left(\frac{\partial^{2} p_{1}(u)}{\partial t^{2}}+(-\Delta+q) p_{1}(u)-a p_{1}(u)-c p_{2}(u), y_{1}(u)\right)_{L^{2}\left(R^{n}\right)} \mathrm{d} t \\
& +\int_{0}^{T}\left(\frac{\partial^{2} p_{2}(u)}{\partial t^{2}}+(-\Delta+q) p_{2}(u)-b p(u)-d p_{2}(u), y_{2}(u)\right)_{L^{2}\left(R^{n}\right)} \mathrm{d} t \\
= & \left(B^{*} p, y\right)_{\left(L^{2}(Q)\right)^{2}} .
\end{aligned}
$$

then

$$
\begin{aligned}
& B^{*} p(u)=B^{*}\left(p_{1}(u), p_{2}(u)\right) \\
& =\left\{\frac{\partial^{2} p_{1}(u)}{\partial t^{2}}+(-\Delta+q) p_{1}(u)-a p_{1}(u)-c p_{2}(u), \frac{\partial^{2} p_{2}(u)}{\partial t^{2}}+(-\Delta+q) p_{2}(u)-b p_{1}(u)-d p_{2}(u)\right\} . \\
& \text { and } A^{*} p(u)=\left(p_{1}(u), p_{2}(u)\right)=\left((-\Delta+q) p_{1}(u)-a p_{1}(u)-c p_{2}(u),(-\Delta+q) p_{2}(u)-b p_{1}(u)-d p_{2}(u)\right)
\end{aligned}
$$


since the adjoint equation takes the form [11]: $\frac{\partial^{2} p(u)}{\partial t^{2}}+A^{*} p(u)=y(u)-z_{d}$

and from theorem (3.1), we have a unique solution $p(u) \in\left(L^{2}\left(0, T ; V_{q}\left(R^{n}\right)\right)\right)^{2}$ which satisfies $p_{1}(u)$, $p_{2}(u) \in L^{2}\left(0, T ; V_{q}\left(R^{n}\right)\right), \frac{\partial p_{1}(u)}{\partial t}, \frac{\partial p_{2}(u)}{\partial t} \in L^{2}(Q)$.

This proves system (15).

Now, we transform (18) by using (15) as follows:

$$
\begin{aligned}
& \int_{0}^{T}\left(\frac{\partial^{2} p_{1}(u)}{\partial t^{2}}+(-\Delta+q) p_{1}(u)-a p_{1}(u)-c p_{2}(u), y_{1}(v)-y_{1}(u)\right)_{L^{2}\left(R^{n}\right)} \mathrm{d} t \\
& \quad+\int_{0}^{T}\left(\frac{\partial^{2} p_{2}(u)}{\partial t^{2}}+(-\Delta+q) p_{2}(u)-b p_{1}(u)-d p_{2}(u), y_{2}(v)-y_{2}(u)\right)_{L^{2}\left(R^{n}\right)} \mathrm{d} t \\
& \quad+(N u, v-u)_{\left(L^{2}(\Sigma)\right)^{2}} \geq 0 .
\end{aligned}
$$

Using Green formula, we obtain:

$$
\begin{aligned}
& \int_{0}^{T}\left(p_{1}(u),\left(\frac{\partial^{2}}{\partial t^{2}}+(-\Delta+q)\right) y_{1}(v)-y_{1}(u)\right)_{L^{2}\left(R^{n}\right)} \mathrm{d} t+\int_{0}^{T}-a\left(p_{1}(u), y_{1}(v)-y_{1}(u)\right)_{L^{2}\left(R^{n}\right)} \mathrm{d} t \\
& \quad+\int_{0}^{T}-c\left(p_{2}(u), y_{1}(v)-y_{1}(u)\right)_{L^{2}\left(R^{n}\right)} \mathrm{d} t+\int_{0}^{T}\left(p_{2}(u),\left(\frac{\partial^{2}}{\partial t^{2}}+(-\Delta+q)\right) y_{2}(v)-y_{2}(u)\right)_{L^{2}\left(R^{n}\right)} \mathrm{d} t \\
& \quad+\int_{0}^{T}-b\left(p_{1}(u), y_{2}(v)-y_{2}(u)\right)_{L^{2}\left(R^{n}\right)} \mathrm{d} t+\int_{0}^{T}-d\left(p_{2}(u), y_{2}(v)-y_{2}(u)\right)_{L^{2}\left(R^{n}\right)} \mathrm{d} t+(N u, v-u)_{\left(L^{2}(\Sigma)\right)^{2}} \geq 0 .
\end{aligned}
$$

Using (12), we have:

$$
(p(u)+N u, v-u)_{\left(L^{2}(\Sigma)\right)^{2}} \geq 0 .
$$

Thus the proof is complete.

\section{Formulation of the Problem When the Observation Is on the Boundary}

The observation equation is given by:

$$
\begin{gathered}
z(u)=\left(z_{1}(u), z_{2}(u)\right)=M\left(\left.y(u)\right|_{\Sigma}\right)=M\left(\left(\left.y_{1}(u)\right|_{\Sigma}\right),\left(\left.y_{2}(u)\right|_{\Sigma}\right)\right) \\
M \in L\left(\left(L^{2}(\Sigma)\right)^{2},\left(L^{2}(\Sigma)\right)^{2}\right) .
\end{gathered}
$$

This is interpreted as follows [11]: we take the trace of $y(u)$ on $\Sigma$, which is particular in $\left(L^{2}(\Sigma)\right)^{2}$. Let this be denoted by $\left.y(u)\right|_{\Sigma}$.

For a given $z_{d}=\left(z_{d 1}, z_{d 2}\right) \in\left(L^{2}(\Sigma)\right)^{2}$, the cost function is given by:

$$
\left.J(v)=\left\|\left.y_{1}(u)\right|_{\Sigma}-z_{d 1}\right\|_{L^{2}(\Sigma)}^{2}+\left\|\left.y_{2}(u)\right|_{\Sigma}-z_{d 2}\right\|_{L^{2}(\Sigma)}^{2}+(N v, v)\right)_{\left(L^{2}(\Sigma)\right)^{2}} .
$$

where $N \in L\left(\left(L^{2}(\Sigma)\right)^{2},\left(L^{2}(\Sigma)\right)^{2}\right)$ is defined as in (14).

The control problem then is to find $u=\left(u_{1}, u_{2}\right) \in U_{a d}$ such that $J(u) \leq J(v)$, where $U_{a d}$ is a closed con- 
vex subset of $\left(L^{2}(\Sigma)\right)^{2}$.

Since the cost function (19) can be written as [11]:

$$
J(v)=a(v, v)-2 L(v)+\left\|y(0)-z_{d}\right\|_{\left(L^{2}(\Sigma)\right)^{2}}^{2},
$$

where $a(v, v)$ is a continuous coercive bilinear form and $L(v)$ is a continuous linear form on $\left(L^{2}(\Sigma)\right)^{2}$. Then using the general theory of Lions [11], there exists a unique optimal control $u \in U_{a d}$ such that $J(u)=\inf J(v)$ for all $v \in U_{a d}$. Moreover, we have the following theorem which gives the necessary and sufficient conditions of optimality:

Theorem (5.1):

Assume that (9) and (14) hold. If the cost function is given by (19), the optimal control $u=\left(u_{1}, u_{2}\right) \in\left(L_{2}(\Sigma)\right)^{2}$ is then characterized by the following equations and inequalities:

$$
\begin{cases}\frac{\partial^{2} p_{1}(u)}{\partial t^{2}}+(-\Delta+q) p_{1}(u)-a p_{1}(u)-c p_{2}(u)=0 & \text { in } Q, \\ \frac{\partial^{2} p_{2}(u)}{\partial t^{2}}+(-\Delta+q) p_{2}(u)-b p_{1}(u)-d p_{2}(u)=0 & \text { in } Q, \\ p_{1}, p_{2} \rightarrow 0 \text { as }|x| \rightarrow \infty, & \text { in } R^{n}, \\ \left.\frac{\partial p_{1}(u)}{\partial v}\right|_{\Sigma}=\left.y_{1}(u)\right|_{\Sigma}-z_{d 1},\left.\quad \frac{\partial p_{2}(u)}{\partial v}\right|_{\Sigma}=\left.y_{2}(u)\right|_{\Sigma}-z_{d 2}, & \text { in } R^{n} . \\ \frac{p_{1}(x, T, u)=p_{2}(x, T, u)=0}{\partial p_{1}(x, T, u)} & \frac{\partial p_{2}(x, T, u)}{\partial t}=0\end{cases}
$$

with $p_{1}(u), p_{2}(u) \in L^{2}\left(0, T ; V_{q}\left(R^{n}\right)\right), \frac{\partial p_{1}(u)}{\partial t}, \frac{\partial p_{2}(u)}{\partial t} \in L^{2}(Q)$ together with (16) and (12).

Proof:

The optimal control $u=\left(u_{1}, u_{2}\right) \in\left(L_{2}(\Sigma)\right)^{2}$ is characterized by [11]:

$$
J^{\prime}(u)(v-u) \geq 0, \quad \forall v \in U_{a d}
$$

Which is equivalent to:

$$
\left(y(u)-z_{d}, y(c)-y(u)\right)_{\left(L^{2}(\Sigma)\right)^{2}}+(N u, v-u)_{\left(L^{2}(\Sigma)\right)^{2}} \geq 0
$$

i.e.

$$
\left(y_{1}(u)-z_{d 1}, y_{1}(v)-y_{1}(u)\right)_{L^{2}(\Gamma)}+\left(y_{2}(v)-z_{d 2}, y_{2}(v)-y_{2}(u)\right)_{L^{2}(\Gamma)}+(N u, v-u)_{\left(L^{2}(\Sigma)\right)^{2}} \geq 0
$$

this inequality can be written as:

$$
\int_{0}^{T}\left[\left(y_{1}(u)-z_{d 1}, y_{1}(v)-y_{1}(u)\right)_{L^{2}(\Gamma)}+\left(y_{2}(v)-z_{d 2}, y_{2}(v)-y_{2}(u)\right)_{L^{2}(\Gamma)}\right] \mathrm{d} t+(N u, v-u)_{\left(L^{2}(\Sigma)\right)^{2}} \geq 0
$$

since the adjoint system takes the form [11]:

$$
\begin{cases}\frac{\partial^{2} p(u)}{\partial t^{2}}+A^{*} p(u)=0 & \text { in } Q \\ \left.\frac{\partial p(u)}{\partial v}\right|_{\Sigma}=y(u)-z_{d} & \text { on } \Sigma\end{cases}
$$


and from theorem (3.1), we get a unique solution $p(u) \in\left(L^{2}\left(0, T ; V_{q}\left(R^{n}\right)\right)\right)^{2}$ which satisfies:

$p_{1}(u), p_{2}(u) \in L^{2}\left(0, T ; V_{q}\left(R^{n}\right)\right), \frac{\partial p_{1}(u)}{\partial t}, \frac{\partial p_{2}(u)}{\partial t} \in L^{2}(Q)$.

This proves system (20).

Now, we transform (22) by using (20) as follows:

$$
\int_{0}^{T}\left(\frac{\partial p_{1}(u)}{\partial v}, y_{1}(v)-y_{1}(u)\right)_{L^{2}(\Gamma)} \mathrm{d} t+\int_{0}^{T}\left(\frac{\partial p_{2}(u)}{\partial v}, y_{2}(v)-y_{2}(u)\right)_{L^{2}(\Gamma)} \mathrm{d} t+(N u, v-u)_{\left(L^{2}(\Sigma)\right)^{2}} \geq 0
$$

Using Green formula, we obtain:

$$
\int_{0}^{T}\left(p_{1}(u), \frac{\partial y_{1}(v)}{\partial v}-\frac{\partial y_{1}(u)}{\partial v}\right)_{L^{2}(\Gamma)} \mathrm{d} t+\int_{0}^{T}\left(p_{2}(u), \frac{\partial y_{2}(v)}{\partial v}-\frac{\partial y_{2}(u)}{\partial v}\right)_{L^{2}(\Gamma)} \mathrm{d} t+(N u, v-u)_{\left(L^{2}(\Sigma)\right)^{2}} \geq 0
$$

Using (12), we have:

$$
\int_{0}^{T}\left(p_{1}(u), v_{1}-u_{1}\right)_{L^{2}(\Gamma)} \mathrm{d} t+\int_{0}^{T}\left(p_{2}(u), v_{2}-u_{2}\right)_{L^{2}(\Gamma)} \mathrm{d} t+(N u, v-u)_{\left(L^{2}(\Sigma)\right)^{2}} \geq 0,
$$

which is equivalent to:

$$
(p(u)+N u, v-u)_{\left(L^{2}(\Sigma)\right)^{2}} \geq 0 .
$$

Thus the proof is complete.

\section{Conclusions}

In this paper, we have some important results. First of all we proved the existence and uniqueness of the state for system (1), which is $(2 \times 2)$ cooperative hyperbolic system involving Schrödinger operator defined on $R^{n}$ (Theorem 3.1). Then we found the necessary and sufficient conditions of optimality for system (1), that give the characterization of optimal control (Theorem 4.1). Finally, we also find the necessary and sufficient conditions of optimal control when the observation is on the boundary (Theorem 5.1).

Also it is evident that by modifying:

-the nature of the control (distributed, boundary),

-the nature of the observation (distributed, boundary),

-the initial differential system,

-the type of equation (elliptic, parabolic and hyperbolic),

-the type of system (non-cooperative, cooperative),

-the order of equation,

many of variations on the above problem are possible to study with the help of Lions formalism.

\section{References}

[1] Serag, H.M. (2000) Distributed Control for Cooperative Systems Governed by Schrödinger Operator. Journal of Discrete Mathematical Sciences and Cryptography, 3, 227-234. http://dx.doi.org/10.1080/09720529.2000.10697910

[2] Bahaa, G.M. (2007) Optimal Control for Cooperative Parabolic Systems Governed by Schrodinger Operator with Control Constraints. IMA Journal of Mathematical Control and Information, 24, 1-12. http://dx.doi.org/10.1093/imamci/dnl001

[3] Qamlo, A.H. (2013) Distributed Control for Cooperative Hyperbolic Systems Involving Schrödinger Operator. International Journal of Dynamics and Control, 1, 54-59. http://dx.doi.org/10.1007/s40435-013-0007-z

[4] Qamlo, A.H. (2013) Optimality Conditions for Parabolic Systems with Variable Coefficients Involving Schrödinger Operators. Journal of King Saud University—Science, 26, 107-112. http://dx.doi.org/10.1016/j.jksus.2013.05.005

[5] Serag, H.M. (2004) Optimal Control of Systems Involving Schrödinger Operators. International Journal of Control and Intelligent Systems, 32, 154-157. http://dx.doi.org/10.2316/Journal.201.2004.3.201-1319

[6] Serag, H.M. and Qamlo, A.H. (2005) On Elliptic Systems Involving Schrödinger Operators. The Mediterranean Jour- 
nal of Measurement and Control, 1, 91-96.

[7] Bahaa, G.M. (2006) Boundary Control for Cooperative Parabolic Systems Governed by Schrodinger Operator. Differential Equations and Control Processes, 1, 79-88.

[8] Bahaa, G.M. and Qamlo, A.H. (2013) Boundary Control for $2 \times 2$ Elliptic Systems with Conjugation Conditions. Intelligent Control and Automation, 4, 280-286. http://dx.doi.org/10.4236/ica.2013.43032

[9] Bahaa, G.M. and Qamlo, A.H. (2013) Boundary Control Problem for Infinite Order Parabolic System with Time Delay and Control Constraints. European Journal of Scientific Research, 104, 392-406.

[10] Serag, H.M. and Qamlo, A.H. (2001) Boundary Control for Non-Cooperative Elliptic Systems. Advances in Modeling \& Analysis, 38, 31-42.

[11] Lions, J.L. (1971) Optimal Control of Systems Governed by Partial Differential Equations. Springer Verlag, Berlin. http://dx.doi.org/10.1007/978-3-642-65024-6

[12] Fleckinger, J. (1981) Estimates of the Number of Eigenvalues for an Operator of Schrödinger Type. Proceedings Royal Society Edinburgh Section A: Mathematics, 89, 355-361.

[13] Fleckinger, J. (1994) Method of Sub-Suber Solutions for Some Elliptic System Defined on $R^{n}$. Preprint UMR MIP. Universite Toulouse 3 . France. 
Scientific Research Publishing (SCIRP) is one of the largest Open Access journal publishers. It is currently publishing more than 200 open access, online, peer-reviewed journals covering a wide range of academic disciplines. SCIRP serves the worldwide academic communities and contributes to the progress and application of science with its publication.

Other selected journals from SCIRP are listed as below. Submit your manuscript to us via either submit@scirp.org or Online Submission Portal.
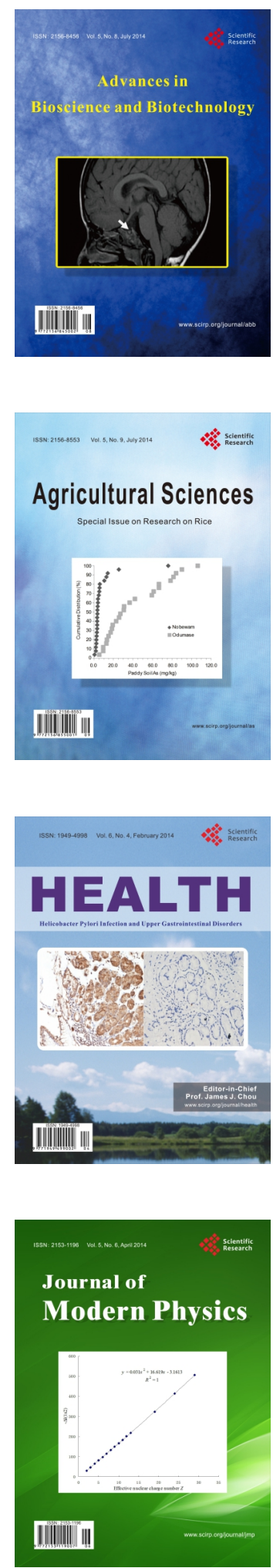
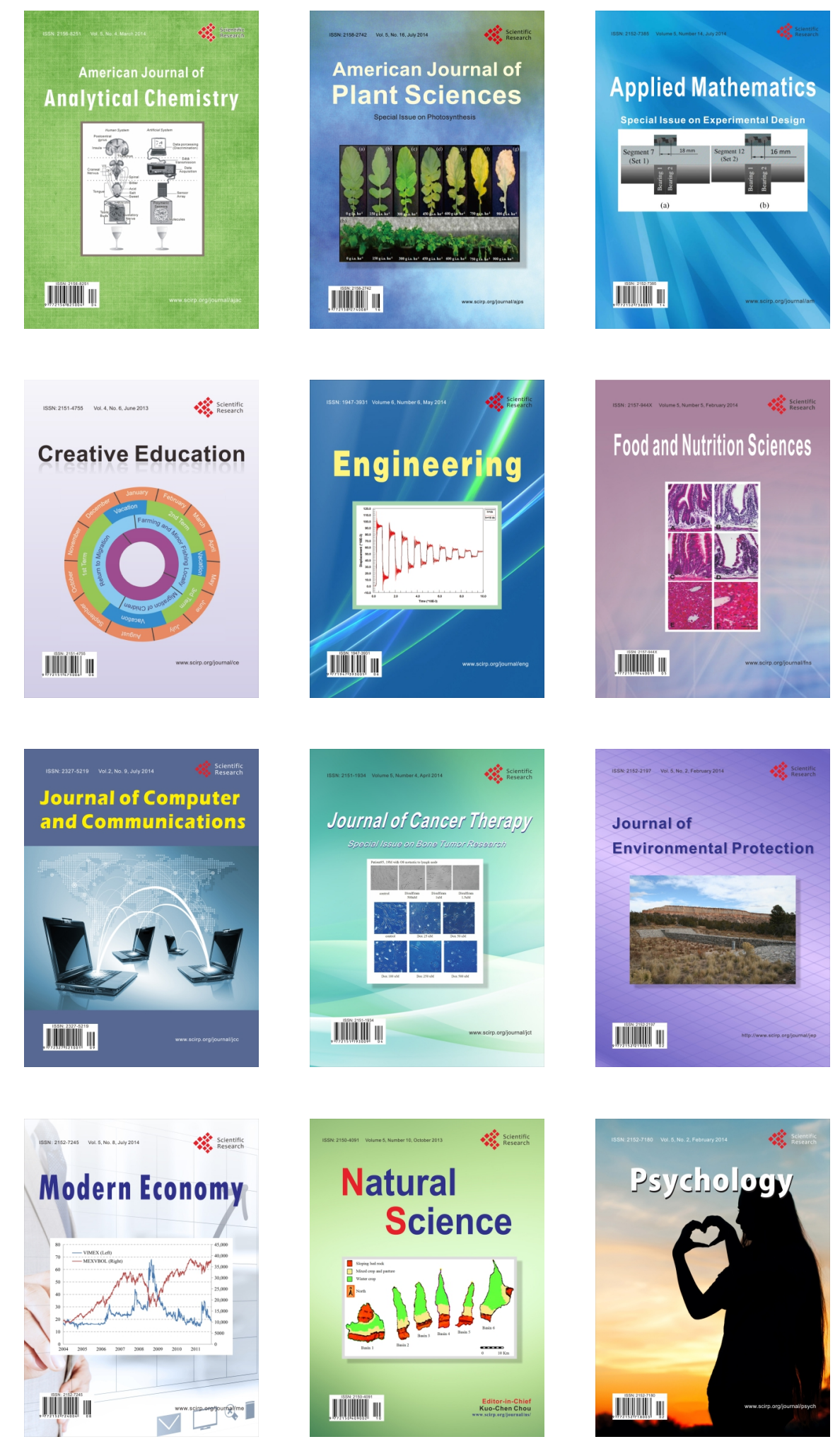